\title{
Low Temperature Resistivity of a-CoFeMnBSi Alloys
}

\author{
S.N. PIRAMANAYAGAM ${ }^{1}$, A.K. NIGAM ${ }^{2}$, G. CHANDRA ${ }^{2}$, S. PRASAD ${ }^{1}$, S.N. SHRINGI ${ }^{1}$, \\ N. VENKATARAMANI ${ }^{3}$, and R. KRISHNAN ${ }^{4}$
}

1. Department of Physics, Indian Institute of Technology, Powai, Bombay - 400 076. India.

2. Low Temperature Physics group, TIFR, Colaba, Bombay - 400005 .

3. Advanced Centre for Research in Electronics, IIT Powai, Bombay - 400 076. India.

4. Laboratoire de Magnetisme et Optique, Versailles, France.

\begin{abstract}
The resistivity below $\mathrm{T}_{m i n}$ of a-Co $\mathrm{Co}_{8-x-y} \mathrm{Fe}_{x} \mathrm{Mn}_{y} \mathrm{~B}_{12} \mathrm{Si}_{8}$ alloys is investigated. The resistivity data was fitted to a logarithmic temperature dependence and the conducitivity data to a $\sqrt{T}$ dependence. $\sqrt{T}$ dependence gave a better fit. From the coefficient of $\sqrt{T}$ term, the diffusion coefficient $\mathcal{D}$ was calculated on the basis of electron-electron interaction effects. $\mathcal{D}$ was found to increase with the concentration of $\mathrm{Mn}$. This also indicated that the density of states at the fermi level increases with the addition of $\mathrm{Mn}$ in these alloys.
\end{abstract}

\section{INTRODUCTION}

The temperature dependence of resistivity $(\rho(\mathrm{T}))$ of amorphous alloys has been a matter of scientific interest for many a researchers in the past. In general, $\rho(\mathrm{T})$ shows a minimum at a temperature $T_{\min }$. This minimum is said to arise from an interplay of two or more scattering mechanisms out of which at least one leading to a negative TCR term and one or more leading to positive TCR term. It is conventional to separate the resistivity behaviour of these alloys into two regions, one in which $\mathrm{T}<\mathrm{T}_{\min }$ and another in which $\mathrm{T}>\mathrm{T}_{\min }$. In general, the number of reports on $\rho(T)<T_{\min }$ is less. Recently, we had reported the resistivity behaviour of a$\mathrm{Co}_{80-x-y} \mathrm{Fe}_{x} \mathrm{Mn}_{y} \mathrm{~B}_{12} \mathrm{Si}_{8}$ alloys for $\mathrm{T}>\mathrm{T}_{\min }$ [1]. In this report, we describe our results on the low temperature resistivity $\left(\mathrm{T}<\mathrm{T}_{\min }\right)$ of $\mathrm{a}_{-} \mathrm{Co}_{80-x-y} \mathrm{Fe}_{x} \mathrm{Mn}_{y} \mathrm{~B}_{12} \mathrm{Si}_{8}$ alloys.

\section{Experimental Details}

The alloys were prepared in the form of ribbons by melt spinning technique. The resistivity was measured by four probe method in the temperature range from $4 \mathrm{~K}$ to $300 \mathrm{~K}$. However, the data used in this report is in the temperature range from $4 \mathrm{~K}$ to $T_{\min }$. The other details are mentioned elsewhere [2].

\section{Theoretical BaCkground}

Attempts have been made in the past to fit the resistivity $\rho(T)$ to a $\ln (T)$ dependence or the conductivity $\sigma(\mathrm{T})$ to a $\sqrt{T}$ dependence, i.e.,

$$
\begin{gathered}
\rho(T)=a-b \ln (T) \\
\sigma(T)=\sigma(0)+d \sqrt{T} \\
1 / R(T)=a 1+b 1 \sqrt{T}
\end{gathered}
$$

These terms correspond to scattering arising from Kondo interaction or from the electron-electron interaction effects.

One of the basic attempts to interpret the resistivity in the negative TCR was to modify and use the Kondo's approach. In Kondo's model, the resistivity arises from the scattering of conduction electrons by randomly isolated spins and follows a logarithmic pattern as in equation 1 . Even though the Kondo model is valid only for metallic alloys with very dilute magnetic impurities, it has been suggested that there could be some spin sites in amorphous alloys which experience a low hyperfine field and hence participate in the Kondo scattering [3].

In high resistivity amorphous alloys, the electron mean free path is comparable to the interatomic distance and hence the electron propagation between collisions is no more classical as there is a significant interference between waves scattered from nearby ions. As a consequence of this, there is a finite probability for an electron to come back to its origin, i.e., a tendency to localization.

The electron-electron interaction effect considers the modification of the effective Coulomb interaction in the presence of a structural disorder. In this case, the temperature dependence of conductivity follows equation 2. 


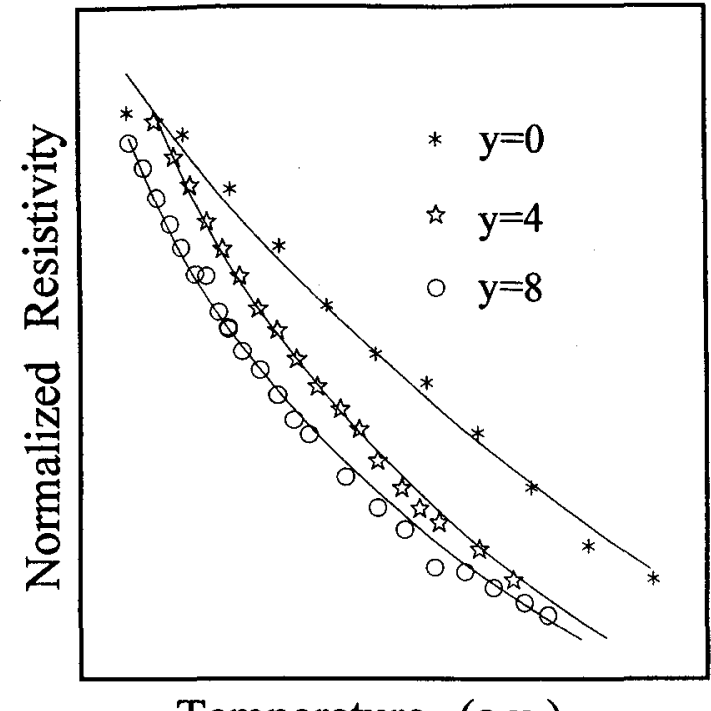

Temperature (a.u.)

Fig. 1. The temperature dependence of resistivity at $\mathrm{T}<\mathrm{T}_{\min }$ and the typical fittings for a-Co80-x-y $\mathrm{Fe}_{x} \mathrm{Mn}_{y} \mathrm{~B}_{12} \mathrm{Si}_{8}$ alloys.

Table I The results of fitting the resistivity data below $\mathrm{T}_{\min }$ of a- $\mathrm{Co}_{80-x-y} \mathrm{Fe}_{x} \mathrm{Mn}_{y} \mathrm{~B}_{12} \mathrm{Si}_{8}$ alloys.

\begin{tabular}{|c|c|c|c|c|c|c|c|}
\hline & & \multicolumn{3}{|c|}{$\mathrm{R}_{n}(\mathrm{~T})=\mathrm{a}-\mathrm{b} \ln (\mathrm{T})$} & \multicolumn{3}{|c|}{$\frac{1}{R_{n}(T)}=a 1+b 1 \sqrt{T}$} \\
\hline $\begin{array}{c}x \\
\text { at\% }\end{array}$ & $\begin{array}{c}\mathrm{y} \\
\text { at\% }\end{array}$ & $\begin{array}{c}a \\
10^{-3}\end{array}$ & $\begin{array}{c}b \\
10^{-4}\end{array}$ & RMSD & a1 & $\begin{array}{c}b 1 \\
10^{-4}\end{array}$ & RMSD \\
\hline 15 & $\overline{0}$ & 1.52 & 5.53 & 0.53 & 1.52 & 6.41 & 0.28 \\
\hline 15 & 4 & 2.31 & 8.01 & 0.53 & 1.82 & 10.6 & 0.49 \\
\hline 15 & 8 & 3.50 & 11.9 & 0.63 & 1.00 & 9.00 & 0.25 \\
\hline 25 & 0 & 1.44 & 6.57 & $\overline{0.43}$ & 2.50 & 13.9 & 0.25 \\
\hline 25 & 4 & 2.34 & 8.11 & 0.62 & 1.13 & 6.80 & 0.34 \\
\hline 25 & 8 & 5.10 & 15.3 & 1.44 & 1.72 & 17.9 & 0.92 \\
\hline 40 & 0 & 1.96 & $6: 89$ & 0.51 & 1.85 & 9.20 & 0.32 \\
\hline 40 & 4 & 2.73 & 9.17 & 0.64 & 1.95 & 12.8 & 0.49 \\
\hline 40 & 8 & 7.20 & 17.4 & 2.23 & 0.60 & 5.99 & 1.60 \\
\hline$\overline{55}$ & $\overline{0}$ & 1.65 & 5.89 & 0.37 & 0.51 & 2.01 & 0.14 \\
\hline 55 & 4 & 1.70 & 7.44 & 0.43 & 0.80 & 4.85 & 0.24 \\
\hline 55 & 8 & 3.62 & 11.9 & 1.63 & 0.70 & 5.98 & 0.66 \\
\hline 70 & $\overline{0}$ & 1.10 & 4.47 & 0.44 & 1.16 & 3.90 & 0.26 \\
\hline 70 & 4 & 1.20 & 5.46 & 0.43 & 1.39 & 9.91 & 0.27 \\
\hline 70 & 8 & 2.00 & 8.50 & 0.49 & 1.51 & 9.96 & 0.40 \\
\hline
\end{tabular}

\section{Results AND Discussion}

Fig.1. shows typical curves of $\rho(\mathrm{T})$ and the fitting to equation 1. The scales of $x$ and $y$ axes are normalized in such a way that the curves for a few Mn concentrations can be shown together. It can be seen that the slope of $y=0$ is less than that of $y=8$.

In order to understand the scattering mechanism in our alloys, we fitted $\rho(\mathrm{T})$ using equation 1 and $1 / \mathrm{R}(\mathrm{T})$ using equation 3 using linear least square method. The value $d$ in equation 2 for our samples was calculated from the coefficient b1 of equation 3 . The results obtained from this fit are given in Table 1 and Table 2.

The following points can be noted from Table 1 .

- Comparison of the RMSD (expressed in 50ppm) inclicate that the $\sqrt{T}$ term fits well for the temper-
Table II The coefficient $\mathrm{d}$ in $\sigma(\mathrm{T})-\sigma(0)=d \sqrt{T}$ and $\mathcal{D}$, the diffusion coefficient of electrons. $d=b 1(A / l)$ where $A$ and $l$ are the area of cross section and the distance between the probes, respectively, during the resistance measurements.

\begin{tabular}{|c|c|c|c|}
\hline $\mathrm{x}$ & $\mathrm{y}$ & $\begin{array}{c}\mathrm{d} \\
(\Omega \mathrm{m})^{-1} \mathrm{~K}^{-\frac{1}{2}}\end{array}$ & $\begin{array}{c}\mathcal{D} \\
\mathrm{m}^{2} \mathrm{~s}^{-1} \\
\times\left(10^{-5}\right)\end{array}$ \\
\hline at\% & at\% & & 7.839 \\
15 & 0 & 309 & 5.184 \\
15 & 8 & 380 & 2.529 \\
\hline 25 & 0 & 454 & 3.584 \\
25 & 4 & 469 & 3.403 \\
25 & 8 & 660 & 1.718 \\
\hline 40 & 0 & 374 & 5.352 \\
40 & 4 & 359 & 5.808 \\
40 & 8 & - & - \\
\hline 55 & 0 & 238 & 13.215 \\
55 & 4 & 267 & 10.500 \\
55 & 8 & 596 & 2.107 \\
\hline 70 & 0 & 237 & 13.327 \\
70 & 4 & 329 & 6.916 \\
70 & 8 & 441 & 3.849 \\
\hline
\end{tabular}

ature dependence of most of the samples. Comparatively, $\ln (T)$ dependence gives a poorer fit.

- The coefficient $b$ of $\ln (T)$ term and $b 1$ of $\sqrt{T}$ in these alloys are of the same order obtained by other workers in similar alloys $[4,5]$.

- The coefficient $b$ increases with the addition of Mn in both Fe-rich and Co-rich alloys.

- The value of $b 1$ also increases with the addition of Mn.

- The increase in the concentration of Fe upto 40 at\% leads to an increase in the coefficient $b$ irrespective of the concentration of $\mathrm{Mn}$. Further addition of $\mathrm{Fe}$ causes a decrease.

- The increase in the concentration of Fe also leads to an increase in the coefficient $d$ irrespective of the concentration of $\mathrm{Mn}$. Beyond $\mathrm{x}=25$, the increase in $x$ leads to a decrease of $d$.

A $\ln (\mathrm{T})$ dependence of resistivity below $\mathrm{T}_{\min }$ has been observed by many other workers in a wide range of amorphous alloys $[2,4,6,7]$. In a-FeNiCrMoSiB alloys, the addition of $\mathrm{Cr}$ increases $\mathrm{b}$ by almost $100 \%$ when $5 \mathrm{at} \%$ of $\mathrm{Cr}$ is added. In general, the substitution of a $3 \mathrm{~d}$ - transition metal impurity increases the coefficient of the $\ln (\mathrm{T})$ term. In our alloys also, the addition of $\mathrm{Mn}$ leads to an increase in the value of $b$, the value of $b$ being maximum for $\mathrm{x}=40$ alloys.

Cochrane and Strom-Olsen [8] have shown that most magnetic as well as non-magnetic metallic glasses follow the empirical rule;

$$
\sigma(T)-\sigma(0)=(500 \pm 100) \sqrt{T}(\Omega m)^{-1}
$$

As indicated in Table 2, the coefficients of $\sqrt{T}$ for our samples also turn out to be of the same order of magnitude as given in equation. Various workers have also 
obtained the coefficient of $\sqrt{T}$ term of the same order as in equation $[5,9]$. From the coefficient of the $\sqrt{T}$ term, one can calculate the value of the diffusion coefficient $\mathcal{D}$ from the equation,

$$
\sigma(T)-\sigma(0)=\frac{1.3}{\sqrt{2}} \frac{e^{2}}{4 \pi^{2} h}\left(\frac{4}{3}-\frac{3}{2} F\right)\left(\frac{2 \pi k_{B} T}{h \mathcal{D}}\right)^{1 / 2}
$$

where e is charge of the electron, $\mathrm{k}$ is the Boltzmann's constant, $h$ is the Plank's constant, $\mathcal{D}$ is the diffusion coefficient and $F$ is the screening factor for Coulomb interaction. Das and Majumdar have calculated $\mathcal{D}$ by assuming $F=0$ in Co-based alloys. We have also calculated the $\mathcal{D}$ values for our samples in the same way and shown them in Table 2. One may notice from Table 2 that the value of $\mathcal{D}$ is of the same order as has been observed by Das and Majumdar. Moreover, the following points can be noted.

- $\mathcal{D}$ decreases with the increase in the concentration of $\mathrm{Mn}$.

- With the addition of Fe, for $y=0$ alloys, it shows an initial decrease and then an increase.

Das and Majumdar have also calculated the density of states at the Fermi level $\left(N\left(E_{F}\right)\right)$ using the relation,

$$
\mathcal{D}=\left(\rho(0) e^{2} N\left(E_{F}\right)\right)^{-1}
$$

One may note that $\mathrm{N}\left(\mathrm{E}_{F}\right)$ and $\mathcal{D}$ are inversely proportional to each other. This equation would indicate that, in all the alloys under present study (irrespective of the Fe concentration), $\mathrm{N}\left(\mathrm{E}_{F}\right)$ increases as the concentration of $\mathrm{Mn}$ increases.

One would, therefore, tend to compare the values of $\mathrm{N}\left(\mathrm{E}_{F}\right)$ obtained from the above equation for our alloys with the values of coefficient of electronic contribution to specific heat capacity $g$, reported for similar alloys, as $\mathrm{g}$ is proportional to the density of states at the Fermi surface. However, it should be pointed out that the values of $N\left(E_{F}\right)$ obtained from this equation have the dimensions of (Volume*energy) while those obtained from the experimental $g$ values have the dimensions of (mole*energy) .

Nevertheless, it is interesting to compare the percentage of change in the density of states of our alloys with those obtained by others. For this purpose, we shall compare the values of $1 / \mathcal{D}$ obtained by us with the g-values obtained by others with the assumption that the number of charge carriers per unit volume does not change mucli with the composition. In Co-based alloys of the present study, an increase in the value of $1 / \mathcal{D}$ of $209 \%$ was observed when the concentration of $M n$ was changed from 0 to 8 . Though an increase in the $\mathrm{g}$ was also observed by Onn et al [10] in a- $\left(\mathrm{Co}_{1-x} \mathrm{Mn}_{x}\right)_{76} \mathrm{~B}_{24}$ alloys, the percentage of increase $(32 \%)$ was smaller compared to that observed in our alloys.
We would like to recall, at this point, that the increase in the slopes observed for $\mathrm{T}<\mathrm{T}_{\min }$ is not specific only to our alloys but was also observed for other early $3 \mathrm{~d}$ - transition metal containing alloy systems also. In $\mathrm{Cr}$ containing alloys also, a similar increase in the slope has been observed. If the present model is applied there, it would mean that in those alloys also, there was an increase in the density of states at $E_{F}$ with this increase of $\mathrm{Cr}$ concentration. It is interesting to note that the value of $\mathrm{g}$ was also found to increase with the addition of $\mathrm{Cr}[11,12]$.

As the concentration of Fe increases, for $y=0$ alloys, we find that the value of $\mathcal{D}$ shows an initial decrease and then an increase indicating that the density of states increases initially (upto $\mathrm{x}=25$ ) and then decreases for higher concentrations of $\mathrm{Fe}$. However, a different result was observed by Kuentzler and Williams [13] for the coefficient of $\mathrm{g}$ in a-FeCoSiB alloys, where the value $g$ was found to show an initial decrease and then an increase, the decrease being maximum for $x=40$. A similar observation to that of Kuentzler and Williams has also been reported by Mizutani et al [12] who observed a minimum in the value of $g$ for for $x=0.6$ in a- $\left(\mathrm{Fe}_{1-x} \mathrm{Co}_{x}\right)_{77} \mathrm{~B}_{13} \mathrm{Si}_{10}$ alloys.

From the above discussions, we tend to conclude that some sort of systematic is observed in the density of states at the Fermi surface if these are evaluated under the assumption that the scattering in the region of $\mathrm{T}$ $<\mathrm{T}_{\min }$ is caused by electron-electron interaction.

\section{REFERENCES}

(1) S.N. Piramanayagam, A.K. Nigam, Shiva Prasad, N. Venkataramani, Girish Chandra, S.N. Shringi, R. Krishnan, J. Appl. Phys. Vol.73[10], p5595 (1993).

[2] A.K. Nigam, Nirupama Sharma, Shiva Prasad, Girish Chandra, S.N. Shringi, R. Krishnan and P. Rougier, J. Ma.gn. Magn. Mater., Vol.102, p297 (1991).

[3] T.E. Sharon and C.C. Tseui, Phys. Rev. B., Vol. 5, p1047 (1972).

[4] G. Rajaram, Girish Chandra, Shiva Prasad, V. Srinivas, S.N. Shringi and R. Krishnan, IEEE Trans. Magn. Vol.20, p1347 (1984).

[5] A. Das and A.K. Majumdar, Phys. Rev. B., Vol.43, p6042 (1991).

[6] H. Gudmundson, H.U. Astrom, D. New, K.V. Rao and H.S. Chen, J. de Phys., Vol.C6, p943 (1978).

(7) E. Babic, Z. Marohnic and J. Ivkov., Solid State Commun. Vol.27, p441 (1978).

18] R.W. Cochrane and J.O. Strom-Olsen, Phys. Rev. B, Vol.29, p1088 (1984).

[9] M. Olivier, J.O. Strom-Olsen, and Z. Altounian, Phys. Rev. B, Vol.35, p333 (1987).

[10] D.G. Onn, Y. Obi and L.Q. Wang, J. Appl. Phys., Vol.53, p7762 (1982).

[11] M. Hasegawa, M. Suzuki and U. Mizutani, IEEE Trans. Magn., Vol.23, P2545 (1987).

[12] U. Mizutani, M. Hasegawa, K. Fukamichi, T. Goto and T. Matsuda, Mater. Trans. JIMI, Vol.30, 953 (1989).

[13] R. Kuentzler and D.E.G. Williams, J. Phys. F., Vol.15, p2283 (1985). 